\title{
Effect of ageing on peak expiratory flow variability and nocturnal exacerbations in bronchial asthma
}

\author{
V. Bellia, G. Cuttitta, F. Cibella, A.M. Vignola, G. Crescimanno, \\ P. D'Accardi, F. Catalano, G. Bonsignore
}

Effect of ageing on peak expiratory flow variability and nocturnal exacerbations in bronchial asthma. V. Bellia, G. Cuttitta, F. Cibella, A.M. Vignola, G. Crescimanno, P. D'Accardi, F. Catalano, G. Bonsignore. CERS Journals Ltd 1997.

ABSTRACT: The aim of this study was to evaluate the role of ageing on variability of airflow obstruction and on the specific pattern of nocturnal exacerbations ("morning dipping" of peak expiratory flow (PEF)) in asthma.

Two groups of stable asthmatics (Group A: 23 patients, aged 14-47 yrs; Group B: 20 patients, aged 53-74 yrs), that were similar for duration of disease, degree of obstruction and response to bronchodilators, were studied. PEF was monitored four times daily for 2 weeks, and amplitude of variation and "morning dip" were calculated.

Both PEF amplitude and "morning dip" were greater in the older patients $(\mathrm{p}<0.005)$. Amplitude was negatively correlated with baseline forced expiratory volume in one second $(F E V 1)$ in both groups $(\mathbf{p}<0.01)$; multiple correlation with age, duration of disease, baseline FEV1 and degree of reversibility was significant in the older group only. Morning dip was inversely correlated to baseline FEV1 $(p<0.01)$ and reversibility $(p<0.05)$. Nocturnal symptoms were reported by all of the five Group A "dippers", but by only 6 of the 13 Group B "dippers" $(\mathbf{p}<0.04)$.

Ageing contributes to increased variability of airway calibre in asthma. Aged patients are potentially at risk because functional evidence of nocturnal asthma may frequently be accompanied by a poor subjective awareness. Therefore, a more extensive practice of PEF monitoring is recommended in aged asthmatics.

Eur Respir J 1997; 10: 1803-1808.
CNR - Istituto di Fisiopatologia Respiratoria and Clinica Pneumologica dell'Università, Palermo, Italy.

Correspondence: V. Bellia

Istituto di Pneumologia

via Trabucco 180

90146 Palermo

Italy

Keywords: Ageing

bronchial asthma

nocturnal asthma

peak expiratory flow

variability

Received: March 71996

Accepted after revision April 141997
In bronchial asthma, the degree of airflow limitation is variable and this variability correlates with the degree of airway responsiveness to bronchoconstrictor stimuli [1]. A particular aspect of this variability is nocturnal asthma. In fact, nocturnal sleep is associated with manifold events and conditions that may induce or facilitate the exacerbation of airway inflammation and obstruction, including: circadian oscillations in the balance of autonomic control of the airways (with increased vagal tone); changes in hormonal levels (with decreased plasma concentrations of epinephrine and cortisol); airway cooling; and exposure to bedding allergens [2]. Therefore, the occurrence of breathlessness, wheezing and cough at night is reported, at least occasionally, by the vast majority of patients [3].

The degree of bronchial hyperresponsiveness is reported to increase with ageing [4]. In addition, some of the factors promoting or facilitating nocturnal attacks (including oscillations in the balance of autonomic control of airway smooth muscle and ventilatory adaptations to resistive load) may undergo changes with ageing. However, the importance of circadian changes in airflow in the aged is not known, since objective assessment of the phenomenon by self-measurement of peak expiratory flow (PEF) rate is not yet a common practice in the older age group.

This study, carried out in stable asthmatics of different ages but with a comparable degree of dysfunction, was aimed at evaluating the role of ageing on the general phenomenon of variability of airflow obstruction and on the specific pattern of nocturnal exacerbation, as expressed by "morning dipping" of PEF.

\section{Subjects and methods}

The investigation was carried out on patients attending an out-patient asthma clinic. Two groups of asthmatic adults of different age were investigated: the younger group (Group A) included 23 patients aged 14-47 yrs (mean age $35 \mathrm{yrs}$ ); the older group (Group B) included 20 patients aged 53-74 yrs (mean age 60 yrs). Table 1 presents their mean anthropometric and clinical characteristics, including: atopic status (expressed in terms of positive response to skin tests); personal history of asthma of $>5$ yrs (early onset) or $\leq 5$ yrs (late onset); and

Table 1. - Characteristics of the groups studied

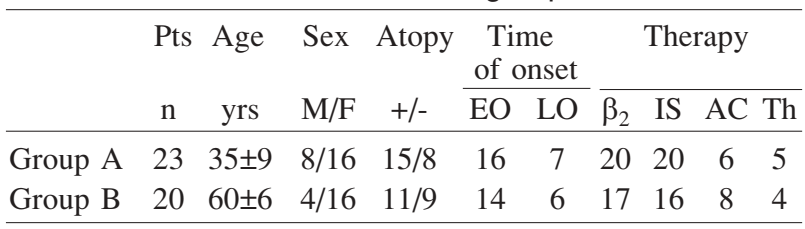

Pts: patients; M: male; F: female; +: positive; -: negative; EO: early onset; LO: late onset; $\beta_{2}: \beta_{2}$-agonists; IS: inhaled steroids; AC: anticholinergic; Th: theophylline. 
medications. All patients were lifelong nonsmokers and free from comorbid conditions; none of them was under treatment with hypnotics, sedatives, or any other kind of drugs able to interfere with the perception of symptoms or the performance of measurements.

Subjects were selected on the basis of the following common criteria: diagnosis of chronic asthma, defined according to American Thoracic Society (ATS) criteria [5]; and stable conditions as expressed by no hospital admission or change in therapeutic regimen in the preceding 3 months.

Subjects were also selected in order that the two groups did not statistically differ according to the following criteria: duration of disease; degree of functional derangement (expressed by forced expiratory volume in one second (FEV1) in \% of predicted) under baseline conditions; and degree of response to treatment over the examined period $(\triangle F E V 1)$, expressed as the ratio of maximum attainable FEV1 over baseline FEV1 per cent.

After providing written informed consent, patients were submitted to spirometry under baseline conditions and $30 \mathrm{~min}$ after inhalation of a bronchodilator by metered aerosol (salbutamol $200 \mu \mathrm{g}$ ). Spirometry was always performed before lunch time, several hours after waking.

Patients were then instructed to record PEF by means of a portable meter (mini-Wright peak flow meter; Clement Clarke Ltd, London, UK). In all cases a careful educational activity on this topic (or reinforcement in cases already familiar with the technique) was provided. The quality of performance was verified at the end of a 2 week period; no difference between groups was noticed in this regard. Measurements were performed four times daily (including early, at the time of getting up in the morning, and late, at the time of going to bed in the evening). PEF was always measured in the standing position in triplicate, and the highest value recorded on a diary chart.

On the same chart, the patients recorded separately daytime and night-time asthma symptoms (cough, shortness of breath and wheeze, by responding to specific questions), as well as the use of short-acting $\beta_{2}$-agonists as rescue medication.

For each patient, the following PEF indices were calculated over $24 \mathrm{~h}$ periods, and then averaged over a 2 week observation period:

1) amplitude of variation, corresponding to:

$$
\frac{\text { maximum value }- \text { minimum value }}{1 / 2(\text { maximum value }- \text { minimum value })} \%
$$

2) "morning dip" corresponding to:

$$
\frac{\text { maximum value - minimum value }}{\text { evening value }}
$$$$
\%
$$

Results were expressed as mean \pm standard deviation. Statistical comparisons between groups were performed by Student's t-test for unpaired data. A p-value equal to or less than 0.05 was regarded as significant. Correlations were evaluated by simple and multiple linear regression analysis. The significance of difference between slopes was checked by the analysis of covariance (ANCOVA).
Finally, the frequency distributions of patterns were evaluated by the Chi-squared method [6].

\section{Results}

According to the selection criteria, the groups did not differ for duration of disease $(13 \pm 11$ yrs in Group A versus $14 \pm 12$ yrs in Group B), and baseline FEV1 (71 \pm 25 versus $68 \pm 24 \%$ pred, respectively). Similarly, no statistically significant difference was found between groups as concerned the degree of response to treatment, in terms of change of FEV1 with respect to baseline level ( $\triangle \mathrm{FEV} 1139 \pm 60$ versus $139 \pm 65 \%$ baseline, respectively), and functional levels attainable after treatment, in terms of $\mathrm{FEV} 1 \%$ pred $(89 \pm 24$ versus $83 \pm 17 \%$ pred, respectively) (fig. 1). Finally, groups did not differ from the point of view of atopic status and distribution between early and late onset.

In both groups, the amplitude of variation and the degree of morning dipping showed an obvious correlation, since the two indices do not represent fully independent variables (fig. 2): however, the $\mathrm{r}^{2}$ value was 0.382 in Group A $(\mathrm{p}<0.002)$ and 0.846 in Group B $(p<0.0001)$, and the slopes were statistically different $(\mathrm{p}<0.05)$.

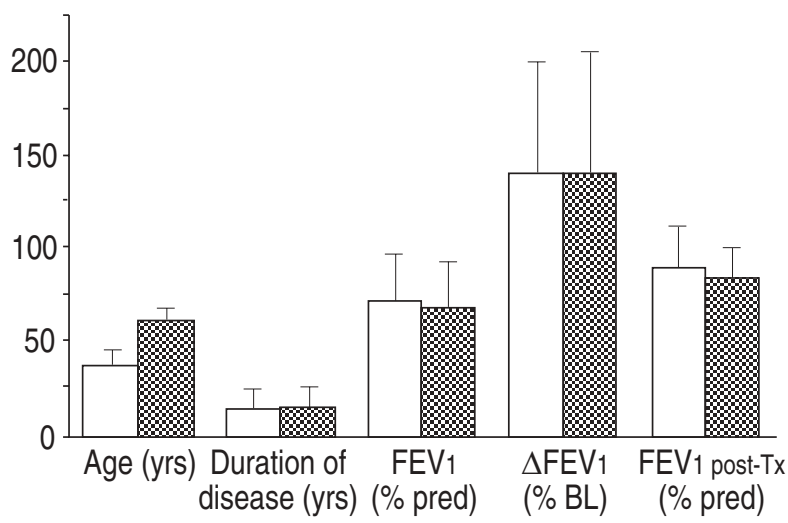

Fig. 1. - Characteristics of the series on the basis of selection criteria. Values are presented as mean \pm sD. $\square$ : Group A; $\$$ : Group B. FEV 1: forced expiratory volume in one second; \% pred: percentage of predicted value; $\triangle \mathrm{FEV}_{1}$ : change in $\mathrm{FEV}_{1}$; \% BL: percentage of baseline value; post-Tx: post-treatment. With the exception of age, none of the indices selected was statistically different between groups.

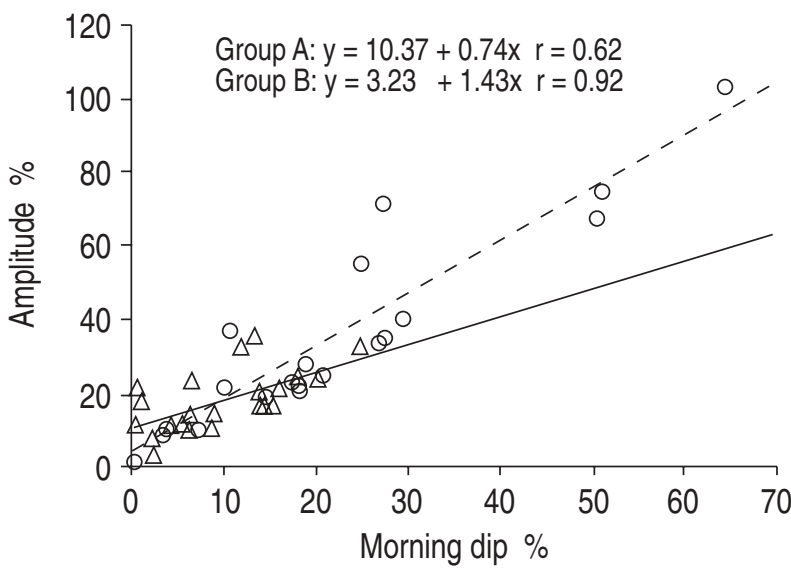

Fig. 2. - Correlation between amplitude of variation and morning dip for the younger patients (Group A; $\Delta$ ) and older patients (Group $\mathrm{B}$; ○). Slopes and difference between slopes were statistically significant. 


\section{Amplitude of variation}

The amplitude of variation was significantly higher in the older age group $(18 \pm 8 \%$ in Group A versus $35 \pm$ $26 \%$ in Group B; p<0.005) (fig. 3a). Conversely, in both groups, no significant difference was observed between early-onset and late-onset subjects.

The index was not correlated with age or duration of disease or the degree of reversibility within the single groups; however, when data from both groups were pooled, a significant positive correlation with age was found $(\mathrm{p}<0.03)$. The amplitude showed a significant negative correlation with baseline FEV1 (\% pred) in both groups $(p<0.01)$; however, the slope of the relationship was steeper in the older group, the difference between slopes being statistically significant (ANCOVA, $\mathrm{p}<0.001$ ) (fig. 4). When age, duration of disease and degree of reversibility were added to baseline FEV1 as independent variables in a multiple regression, the correlation with amplitude was significant in the older group only; however, $\mathrm{r}^{2}$ increased in both groups (in Group A from 0.190 for simple regression with FEV1, to 0.289 for multiple regression; in Group B from 0.411 to 0.460 ).

\section{Morning dip}

The degree of morning fall in PEF differed significantly in the two groups: its mean value over 2 weeks was $10 \pm 7 \%$ in Group A and $22 \pm 17 \%$ in Group B ( $<<$ 0.005 ) (fig. 3b). Conversely, in both groups the earlyonset cases did not differ significantly from the lateonset ones.
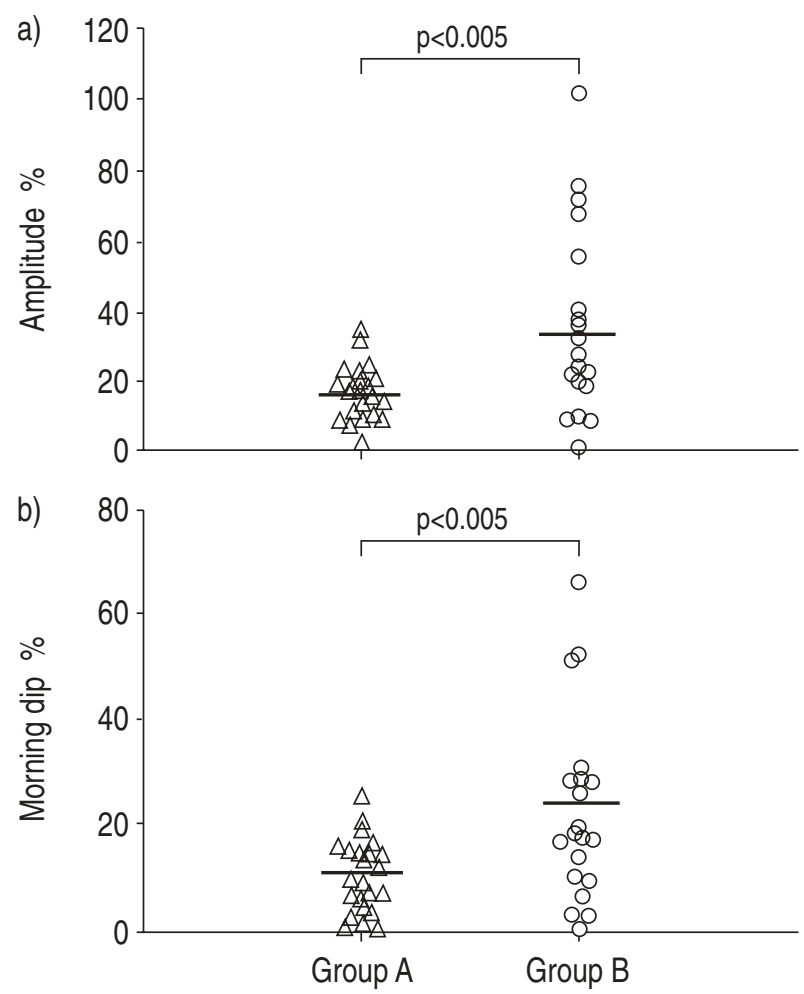

Fig. 3. - Mean individual values for: a) amplitude of variation; and b) morning dip. The horizontal bars indicate the mean group values. The differences between means were significant both for amplitude and morning dip. Group A: younger patients; Group B: older patients.
For the simple regression analysis on pooled data of the two groups, age correlated with the degree of dipping $(\mathrm{p}<0.03)$; conversely, within the younger group the index was not correlated to age, duration of disease, baseline FEV1 or degree of functional reversibility. In the older group, morning dip was inversely correlated to baseline FEV1 $\left(\mathrm{r}^{2}=0.335 ; \mathrm{p}<0.01\right)$ and reversibility $\left(\mathrm{r}^{2}=\right.$ $0.199 ; \mathrm{p}<0.05)$, but not to age or duration of disease. The multiple regression analysis (where age, duration, baseline FEV1 and reversibility were used as independent variables, and morning dip as the dependent variable) did not show a significant result in Group A; conversely, in Group B the p-value approached statistical significance $(0.055)$, with an increase in $\mathrm{r}^{2}(0.44)$ with respect to the simple regression model.

The number of "dippers" (defined as cases whose mean morning dip was equal to, or exceeded, a threshold value of $15 \%$ ) was 5 out of 23 in Group A, as compared to 13 out of 20 in Group B, the difference being

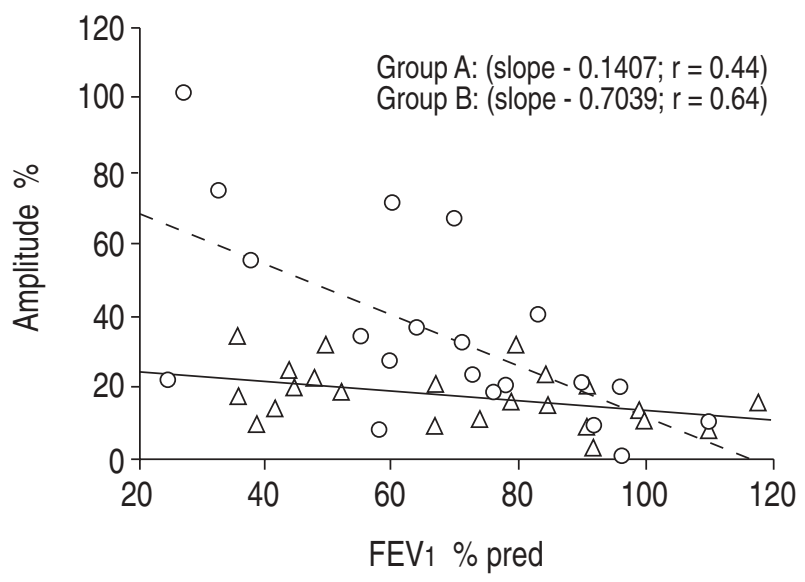

Fig. 4. - Correlation between the baseline level in forced expiratory volume in one second $\left(\mathrm{FEV}_{1}\right)$ and the amplitude of variation in peak expiratory flow (PEF) in the younger patients (Group $\mathrm{A} ;-\square-$ and the older patients (Group B; - - O- - ). The difference between slopes is statistically significant, showing a higher variability in Group $\mathrm{B}$ for lower FEV1 values.

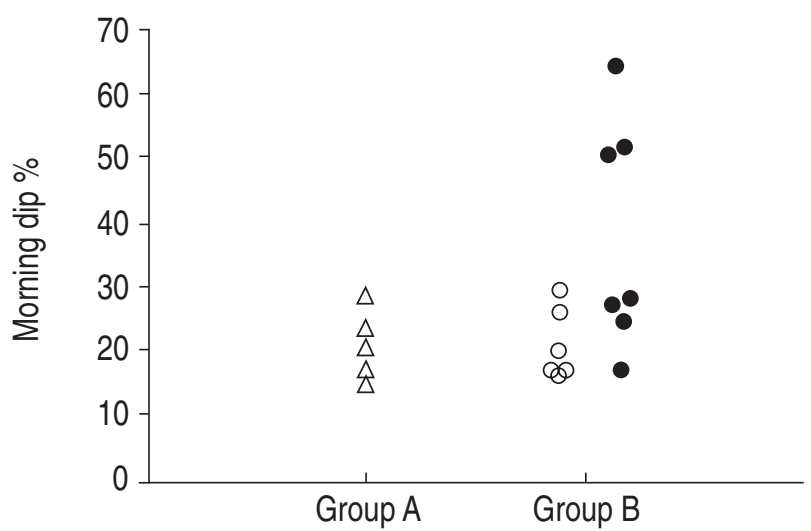

Fig. 5. - Subjective awareness of nocturnal event and percentage of morning dip (only for patients with mean morning dip $>15 \%$ ) in younger patients (Group A; $\Delta$ ) and older patients (Group B; O,৫). Open symbols refer to the self-reported account of nocturnal symptoms; closed symbols indicate lack of subjective awareness. All subjects from Group A with mean morning dip $>15 \%$ reported nocturnal symptoms, while only 6 out of 13 subjects from Group B had subjective awareness of such symptoms. 
significant (Chi-squared, $\mathrm{p}<0.005)$. However, nocturnal symptoms were reported by all of the five Group A "dippers", but in only 6 of the 13 Group B "dippers" (Chi-squared, $\mathrm{p}<0.04$ ); the group lacking subjective awareness included the cases with the most pronounced functional deterioration (fig. 5).

\section{Discussion}

The results of the present study indicate that in adult asthmatics, ageing is associated with an increased variability of airway calibre. As a consequence, independent of the duration of disease and the overall degree of airway dysfunction, as shown by spirometry and reversibility test, a more prominent circadian variability is shown by older patients. This difference cannot be attributed to any recognizable disease characteristic: in fact, the two groups under investigation did not show any significant difference in terms of average severity, medications, atopic status, or early onset. In particular, from the comparison within groups between patients with a history of early onset and those with late onset, no significant difference in variability of PEF was found.

The increased variability of PEF is paralleled by a higher frequency and degree of morning dipping. This was expected, as the amplitude of variation and morning dipping are not independent variables. However, the two parameters are not fully superimposable, as in asthmatics the degree of airway patency is not only under the influence of nocturnal factors, but it may change markedly over daytime hours due to entirely different factors (outdoor environment, physical activity, emotional factors, etc.). In a recent paper, D'Alonzo et al. [7] demonstrated that measurements of PEF twice daily (e.g. morning and evening) may grossly underestimate the circadian variability of PEF. This was confirmed in the present series, where the amplitude of variation (calculated over four values daily) was higher than the corresponding morning dip in all cases. In addition, the data indicate that ageing may affect the two parameters to a different extent, as suggested by the different $r$ and slope values of their correlation recorded in the two age groups.

\section{Variability of airway obstruction}

An increased intra-individual variability of PEF is commonly assumed to be related to instability of bronchomotor tone; as such, it is considered to be a reliable indicator of bronchial hyperresponsiveness, particularly suitable for epidemiological studies [8]. For this reason, International Guidelines on the management of asthma include PEF variability among the parameters suitable for assessment of the severity of the disease [9].

As concerns older subjects, very few investigations have applied the technique of PEF monitoring to this specific setting, and no study on the diagnostic power of PEF in elderly asthmatics has been published so far. In investigations carried out on random population samples, variability has been found to increase with age in the range 18-73 yrs [8] and 20-70 yrs [10]. However, in a recent study on healthy volunteers aged 4-84 yrs, data concerning intra-individual variability in the subset aged over 65 yrs (29 subjects) showed coefficients of variation $(2.7 \%$ in males and $2.0 \%$ in females $)$ not far from the mean value of the general adult population of the same study (2.1\%) [11].

In the present investigation, the significant correlation of variability with baseline FEV1 in both groups confirms previous observations carried out by BAHOUS et al. [12] in young adults with mild airway hyperresponsiveness. Since variability of PEF is an index of bronchial lability, the correlation cited may be interpreted as an effect of airway geometry, in fact, it may be assumed to represent an application of the general concept that the narrower the baseline calibre the higher the percentage response to bronchoconstrictive stimuli, airway resistance being inversely proportional to the fourth power of radius. Interestingly, the slope of the regression line was much steeper in the elderly, the difference being statistically significant, in other words, for an equal level of airway dysfunction, the variability of airway calibre over the day is increased in older patients. This suggests that the airways may become more twitchy with ageing. This finding is in agreement with the observations of Hopp et al. [4], who demonstrated that in healthy subjects aged over 60 yrs airway responsiveness to methacholine increases with age.

An important factor possibly contributing to this agerelated increase in airway responsiveness is the mechanical interdependence between airways and parenchymal structures, as suggested by the results of NAGASE $e t$ al. [13] in an experimental model of ageing rats. In fact, the loss of lung elastic recoil associated with ageing [14] may reduce the effects of parenchymal attachments in preventing an excessive airway narrowing; in addition, ageing may be associated with a change in stiffness of the airway wall. The question is open and warrants further structural and functional studies.

\section{Nocturnal asthma}

The degree of morning fall in PEF has been demonstrated to represent a reliable quantitative estimate of nocturnal exacerbation of airflow obstruction [15]. In the present study, we observed a higher frequency and magnitude of morning dipping in older patients; this finding may be interpreted, in part, as an expression of the age-related increase in reactivity [4]. However, as previously emphasized, factors promoting overall circadian variability may be more numerous and heterogeneous than those producing the functional deterioration at night. This is confirmed by the different result of the multiple regression analysis with age, duration of disease, baseline FEV1 and degree of reversibility as independent variables. In fact, as concerns younger patients, morning dipping, whatever its severity, cannot be explained by these time-related and function-related variables, as demonstrated by the very low $\mathrm{r}^{2}$ value $(0.057)$. Conversely, in the older age group, where some nocturnal exacerbation of airway obstruction affected the majority of patients, the degree of dipping correlated both with the baseline function and the degree of reversibility. In addition, the inclusion of age and duration of disease in the panel of functional indices in a multiple regression model, resulted in a p-value approaching statistical significance, with nearly half of the variance accounted 
for by the selected independent variables. These results, as opposed to those relevant to younger subjects, indicate that the host of factors influencing the parameters investigated may change with ageing.

As mentioned above, some imbalance in the mechanisms of autonomic control of airway smooth muscle activity is certainly involved in the pathogenesis of nocturnal attacks. In this context, the role of the parasympathetic nervous system in determining the normal circadian variations of airway calibre [16], as well as the pathogenesis of nocturnal asthma [17-19], is clearly established. In this perspective, ageing may be expected to have a modulating role. In fact, the process of normal ageing is accompanied by changes in the relative contribution of excitatory and inhibitory afferents to bronchial smooth muscle, with an overall loss of sensitivity affecting, to a proportionally greater extent, the adrenergic component [20]. On this basis, the hypothesis of an agerelated dysfunction of $\beta_{2}$-adrenoceptor has been proposed [21]; the consequences may be particularly evident at night, since a nocturnal decrease in the number and affinity of these receptors has been reported in asthmatics [22].

Another clinically relevant finding of the present study is the lack of awareness of nocturnal deterioration of airway function noticed in the majority of elderly patients showing a definite morning dip of PEF, at variance with observations in the younger age sample. This result is in agreement with previous evidence concerning the reduced perception of load in the elderly. In fact, TACK et al. [23] observed a blunted sensitivity to added inspiratory and expiratory loads in healthy subjects over 60 yrs of age, in spite of a preserved force-generating capability during breathing; This reduction in sensitivity has since been confirmed with respect both to mechanical [24] and chemical loads [25, 26]. The explanation of this phenomenon is still under debate, but may reflect changes in central integration of afferent signals, rather than a defect in the perception of loads [27]. In addition, it must be noted that the elderly are particularly prone to comorbid conditions that may further attenuate the perception of load. Among them, diabetes mellitus is particularly important because of its prevalence. O'Donnell et al. [28] have demonstrated that patients with insulin-dependent diabetes may have an impaired ability to perceive inspiratory-resistive loads.

This condition results in a better tolerance to breathlessness for any given level of obstruction and, possibly, in a less affected quality of life. However, it implies two negative consequences: firstly, it impairs the ability to react to increased load; and secondly, it limits the adaptive changes aimed at preserving the ventilation. As concerns the former, the increased threshold for the perception of respiratory sensations may lead to a delayed recognition of the exacerbation of airway obstruction; this, in turn, would delay the self-administration of bronchodilator medications or the search for medical advice. This is particularly true during nocturnal sleep, which by itself attenuates all of the responses to respiratory stimuli. In a previous study we demonstrated that significant increase in pulmonary resistance may occur during uninterrupted nocturnal sleep, leading to spontaneous remission without awakening [29]. For this reason, as observed in the present study, the patients, and in particular the elderly, may be unaware of the nocturnal event; consequently, accounts based on self-report may substantially underestimate the prevalence and severity of the phenomenon. As a consequence, during nocturnal attacks, elderly asthmatics would be at a particularly high risk of deterioration of airway function.

The other risk of reduced sensitivity is connected with a reduced capacity to adopt strategies aimed at preserving minute ventilation. It has been demonstrated that patients showing a blunted perception of dyspnoea are predisposed to a higher risk of fatal asthma attacks [30]. This may be particularly dangerous in the setting of nocturnal asthma, since it has been demonstrated that during nocturnal attacks ventilatory adaptation to increased resistive loads is highly variable among patients, and that a subset of patients shows an ineffective strategy of adjustment of ventilatory timing and muscular output [31].

In conclusion, in consideration of the age-related increasing frequency of nocturnal exacerbations and the higher risks associated with these events, the practice of peak expiratory flow measurement aimed at monitoring airway obstruction is highly recommended in older asthmatics.

\section{References}

1. Lebowitz M. The use of peak expiratory flow rate measurements in respiratory disease. State of the art review. Pediatr Pulmon 1991; 11: 166-174.

2. Martin R. Nocturnal asthma: an overview. In: Martin R, ed. Nocturnal asthma: Mechanisms and Treatment. Mount Kisco, NY, Futura Publishing, 1993; pp. 71-115.

3. Turner-Warwick M. Epidemiology of nocturnal asthma. Am J Med 1988; 85 (Suppl. 1B): 6-9.

4. Hopp RJ, Bewtra A, Nair NM, Townley RG. The effect of age on methacholine response. J Allergy Clin Immunol 1985; 76: 609-613.

5. ATS. Standards for the diagnosis and care of patients with chronic obstructive pulmonary disease (COPD) and asthma. Am Rev Respir Dis 1987; 136: 225-244.

6. Snedecor G, Cochrane W. Statistical methods. 7th Edn. Ames, Iowa State University Press, 1980.

7. D'Alonzo G, Steinijans V, Keller A. Measurements of morning and evening airflow grossly underestimate the circadian variability of FEV1 and peak expiratory flow rate in asthma. Am J Respir Crit Care Med 1995; 152: 1097-1099.

8. Higgins B, Britton J, Chinn S, Cooper S, Burney P, Tattersfield A. Comparison of bronchial reactivity and peak expiratory flow variability measurements for epidemiologic studies. Am Rev Respir Dis 1992; 145: 588-593.

9. Sheffer AL, ed. Global strategy for asthma management and prevention. NHLBI/WHO Workshop Report. NIH Publication No. 95-3659, 1995.

10. Boezen H, Schouten J, Postma D, Rijcken B. Distribution of peak expiratory flow variability by age, gender and smoking habits in a random population sample aged 20-70 yrs. Eur Respir J 1994; 7: 1814-1820.

11. Hegewald M, Crapo R, Jensen R. Intraindividual peak flow variability. Chest 1995; 107: 156-161.

12. Bahous J, Cartier A, Malo J. Monitoring of peak expiratory flow rates in subjects with mild airway hyperexcitability. Bull Eur Physiopathol Respir 1985; 21: 25-30. 
13. Nagase T, Fukuchi Y, Teramoto S, Matsuse T, Orimo $H$. Mechanical interdependence in relation to age: effects of lung volume on airway resistance in rats. $J$ Appl Physiol 1994; 77: 1172-1177.

14. Turner J, Mead J, Wohl M. Elasticity of human lungs in relation to age. $J$ Appl Physiol 1968; 25: 664-671.

15. Bellia V, Visconti A, Insalaco G, Cuttitta G, Ferrara G, Bonsignore G. Validation of morning dip of peak expiratory flow as an indicator of the severity of nocturnal asthma. Chest 1988; 108: 108-110.

16. Dreher D, Koller E. Circadian rhythms of specific airway conductance and bronchial reactivity to histamine: the effects of parasympathetic blockade. Eur Respir $J$ 1990; 3: 414-420.

17. Catterall J, Rhind G, Whyte K, Shapiro C, Douglas N. Is nocturnal asthma caused by changes in airway cholinergic activity? Thorax 1988; 43: 720-724.

18. Bellia V, Ferrara G, Cibella F, et al. Comparison of the effects of oxitropium bromide and of slow-release theophylline on nocturnal asthma. Postgrad Med J 1988; 64: 583-586.

19. Morrison J, Pearson S. The parasympathetic nervous system and the diurnal variation of lung mechanics in asthma. Respir Med 1991; 85: 285-289.

20. Vestal R, Wood A, Shand D. Reduced $\beta$-adrenoceptor sensitivity in the elderly. Clin Pharmacol Ther 1979; 26: 181-186.

21. Connolly MJ. Ageing, late-onset asthma and the betaadrenoceptor. Pharmacol Ther 1993; 60: 389-404.

22. Titinchi S, Shamma MA, Patel K, Clark B. Circadian variation in number and affinity of $\beta_{2}$-adrenoceptors in lymphocytes of asthmatic patients. Clin Sci 1984; 66: 323-328.
23. Tack M, Altose M, Cherniack N. Effect of aging on the perception of resistive ventilatory loads. Am Rev Respir Dis 1982; 126: 463-467.

24. Connolly M, Crowley J, Charan N, Nielson C, Vestal $\mathrm{R}$. Reduced subjective awareness of bronchoconstriction provoked by methacholine in elderly asthmatic and normal subjects as measured on a simple awareness scale. Thorax 1992; 47: 410-413.

25. Kronenberg R, Drage C. Attenuation of the ventilatory and heart rate response to hypoxia and hypercapnia with aging in normal man. J Clin Invest 1973; 52: 1812-1819.

26. Brischetto M, Millman R, Petersen D, Silage D, Pack A. Effect of aging on ventilatory response to exercise and $\mathrm{CO}_{2} . J$ Appl Physiol: Respirat Environ Exercise Physiol 1984; 56: 1143-1150.

27. Tack M, Altose M, Cherniack N. Effect of aging on sensation of respiratory force and displacement. J Appl Physiol 1983; 55: 1433-1440.

28. O'Donnell C, Friedman L, Russomanno J, Rose R. Diminished perception of inspiratory-resistive loads in insulin-dependent diabetics. N Engl J Med 1988; 319: 1369-1373.

29. Bellia V, Cuttitta G, Insalaco G, Visconti A, Bonsignore G. Relationship of nocturnal bronchoconstriction to sleep stages. Am Rev Respir Dis 1989; 140: 363-367.

30. Kikuchi Y, Okabe S, Tamura G, et al. Chemosensitivity and perception of dyspnea in patients with a history of near-fatal asthma. N Engl J Med 1994; 330: 1329-1334.

31. Cibella F, Cuttitta G, Romano S, Grassino A, Bellia V. Ventilatory response to spontaneous resistive load variations during sleep. J Appl Physiol 1994; 76: 2394 2404. 